\title{
A UV Picture of a Loop Induced Neutrino Mass Model and Its Phenomenological Consequences
}

\author{
Tetsuo Shindou* \\ Division of Liberal-Arts, Kogakuin University, Tokyo, Japan
}

In this article, we review several models where tiny neutrino masses are radiatively generated via loop diagrams. In such models, additional scalar fields are often introduced so that the Standard Model Higgs sector is extended.Such an extension results in a rich phenomenology of the model. We briefly discuss such a model and its UV completion to highlight some of its phenomenological consequences.

Keywords: neutrino mass, extended Higgs sector, UV theory, SUSY model, collider phenomenology

OPEN ACCESS

Edited by:

Stefano Moretti,

University of Southampton,

United Kingdom

Reviewed by:

Chandan Hati,

UMR6533 Laboratoire de Physique de Clermont (LPC), France

Frank Franz Deppisch, University College London,

United Kingdom

*Correspondence:

Tetsuo Shindou

shindou@cc.kogakuin.ac.jp

Specialty section:

This article was submitted to

High-Energy and Astroparticle

Physics,

a section of the journal

Frontiers in Physics

Received: 10 October 2018 Accepted: 20 December 2018

Published: 14 January 2019

Citation:

Shindou T (2019) A UV Picture of a

Loop Induced Neutrino Mass Model and Its Phenomenological

Consequences. Front. Phys. 6:159.

doi: 10.3389/fphy.2018.00159

\section{INTRODUCTION}

Precise measurement of the Higgs boson property at the LHC experiments [1-6] suggests that the Standard Model (SM) provides quite a good explanation of the physics of elementary particles. However, there still are several unsolved problems in the SM. For example, there is no dark matter (DM) candidate, no successful baryogenesis scenario works, gauge hierarchy problems should be solved by some additional mechanism, and so on. An origin of tiny neutrino mass has been one of such problems for more than two decades. The neutrino oscillation data [7-12] requires that there are tiny mass squared differences among three neutrino mass eigenvalues, and the absolute value of the neutrino masses have quite a severe upper bound of $m_{v} \lesssim \mathcal{O}(0.1) \mathrm{eV}[13,14]$.

In many models, the tiny neutrino masses are originated from the dimension five operator $\left(H \cdot \bar{\ell}^{c}\right)(H \cdot \ell)[15]$ after the electroweak symmetry breaking. The question is how to provide the suppressed coefficient of the operator. There are essentially three possibilities to get such a suppression factor naturally. One idea is using a suppression by a mass scale. Since the operator is dimension five, the coefficient is suppressed by some mass scale. If such a mass scale is significantly larger than the electroweak scale, the coefficient of the dimension five operator gets a strong suppression. The necessary mass scale $M$ in this case is naively estimated by the relation $\langle H\rangle^{2} / M \sim$ $m_{v}$, so that $m_{v} \sim 0.1 \mathrm{eV}$ suggests $M \sim 10^{15} \mathrm{GeV}$. The most famous mechanism of this possibility is so-called type I seesaw model [16-20], where heavy right handed neutrinos (RHNs) are introduced to the SM and the dimension five operator is suppressed by this heavy mass scale after decoupling of the RHNs. The second mechanism is that the smallness of the coefficient is naturally explained as a result of slightly broken symmetry. This idea is realized e.g., in inverse seesaw mechanism [21, 22]. The third possibility is that the operator is generated through quantum loop effect [23-34]. In this case, the suppression comes from the loop factor. For example, in a one-loop model, the coefficient gets a suppression factor of $1 /(4 \pi)^{2}$ in addition to a suppression by a particle mass in the loop. In Figure 1, examples of relevant diagrams for neutrino masses are shown in several models. A recent comprehensive review on the third possibility can be found, for example, in Cai et al. [35].

Comparing to the first cases (e.g., type-I seesaw mechanism), one can find that the mass scale of new particles should be much lower in the second cases. In a case that the 
neutrino mass is induced via $n$-loop diagram, the neutrino mass can be roughly estimated as

$$
m_{v} \sim\left(\frac{\lambda^{2}}{(4 \pi)^{2}}\right)^{n} \frac{\langle H\rangle^{2}}{M}
$$

where $\lambda$ is some coupling constant, and $M$ is a mass scale of new particle running in the loop. For example, in a 3-loop model with $\lambda \sim 0.1$, a new particle with a mass $M \sim \mathcal{O}(100 \mathrm{GeV})$ is necessary. Such a new particle can be discovered by future collider experiments such as LHC.

In models where colored new particles run in the loop diagrams for the neutrino masses, these particles can also contribute to several processes in B physics [36-40]. By these new contributions, one can give an explanation of $\mathrm{B}$ anomalies reported by the BaBar experiment and the LHCb experiment [4147]. From this viewpoint, models with loop induced neutrino masses have been attracting a lot of attention.

However, in many cases, such models are constructed as a phenomenological model. We strongly expect that there is a UV complete theory above a cutoff scale as a more fundamental picture of such a phenomenological model. There are a few attempts to construct such a UV picture. For example, in Doršner et al. [48], a grand unified model which leads to loop induced neutrino masses at a low energy scale is proposed. In this article, we introduce another possibility based on SUSY gauge theory with confinement [49-52]. In the low energy effective theory of this theory, the Higgs sector is extended to include necessary fields to draw loop diagrams which leads to the dimension five operator. In the model, DM candidates are included, and the electroweak phase transition is enhanced strongly enough for successful electroweak baryogenesis [53-61].

This review is organized as follows. In section 2, we introduce typical concrete examples of models with loop induced neutrino masses. In section 3, we discuss an example UV picture of such a phenomenological model. We there also discuss phenomenological consequences of the UV theory. A summary is presented in section 4 .

\section{RADIATIVE NEUTRINO MASS MODELS}

In this section, we review typical examples of models with loop induced neutrino mass. The models are classified into two groups. In a class of models with RHNs, there should be an additional symmetry, which is a discrete symmetry in many cases, and the RHNs have a charge under that symmetry. For example, in a model with $Z_{2}$ parity, odd parity is assigned to the RHNs, since the tree level Yukawa coupling of RHNs with the lepton doublets should be forbidden. In another class of models, no RHNs are introduced.

In this review, we focus on models with RHNs [28-34], because such models has a big advantage, which is that there is a DM candidate. In order to generate the dimension five operator, the lepton number should be broken in the loop. In a model with RHNs, the Majorana mass of each RHN breaks the lepton number. As already described, a new symmetry is necessary to forbid the tree level Yukawa couplings of RHNs. To realize this with keeping the Majorana mass term of RHNs, the simplest symmetry is a $Z_{2}$ parity and the odd parity is assigned to the RHNs. In this setup, the lightest neutral $Z_{2}$-odd particle in the model can be a DM candidate, unless the $Z_{2}$ is broken.

A very well-known example of such models with one-loop induced neutrino mass is the Ma model [29], where the $Z_{2}$ odd inert doublet scalar $\eta$ and three $Z_{2}$ odd RHNs $N_{i}$ are introduced to the SM. The dimension five operator is generated via the oneloop diagram shown in Figure 1A. In this model, the lighter one among $N_{i}$ and the neutral component of $\eta$ can be a DM candidate.

Two-loop models with RHNs are also discussed e.g., in Aoki et al. [62], Kajiyama et al. [63]. In the model proposed in Aoki et al. [62], the vertex corresponding to the $\eta \eta H H$ coupling in the Ma model is induced by one-loop. On the other hand, In the model proposed in Kajiyama et al. [63], the Majorana mass terms of $N_{i}$ in the Ma-model are induced by one-loop.

There are examples of three loop models. Let us here introduce two examples. One is called Kraus-Nasri-Trodden model (KNT model) [28], and the other is called Aoki-Kanemura-Seto model (AKS model) [32-34]. In the KNT model, in addition to three $Z_{2}$ odd RHNs, a $Z_{2}$ even singly (electric) charged singlet scalar $\omega_{1}^{-}$and a $Z_{2}$ odd singly charged singlet scalar $\omega_{2}^{-}$are introduced. The three loop diagram for the dimension five operator is shown in Figure 1B.

In the AKS model, the discrete symmetry $Z_{2} \times Z_{2}^{\prime}$ is imposed. The $Z_{2}$ parity is assumed to be unbroken, while the $Z_{2}^{\prime}$ symmetry is softly broken in the Lagrangian. For the particle content, an extra scalar doublet $H^{\prime}$, three RHNs $N_{i}$, a neutral singlet scalar $\zeta$, and a charged singlet scalar $\Omega^{-}$are introduced to the SM. Under the $Z_{2} \times Z_{2}^{\prime}$, the $S M$ particles and the new particles are charged as $q(+,+), u_{R}(+,-), d_{R}(+,-), \ell(+,+), e_{R}(+,+)$, $H(+,+), H^{\prime}(+,-), \Omega^{-}(-,+), \zeta(-,-)$, and $N_{i}(-,+)$. With this parity assignment, the Higgs sector of the model is nothing but the Type-X two Higgs doublet model [64]. The neutrino masses are generated by the three loop diagram shown in Figure 1C. In this model, the unbroken $Z_{2}$ symmetry guarantees the stability of the DM, so that the lightest neutral particle among $N_{i}$ and $\zeta$ can be a DM candidate. In addition, it is nice that the electroweak phase transition is enhanced by loop contributions of $Z_{2}$-odd particles in this model. As mentioned later, such enhancement of electroweak phase transition is required for successful electroweak baryogenesis. Therefore, the AKS models has a potential to solve three big problems in the SM, neutrino mass, DM, and baryogenesis.

SUSY extension of these models are also discussed in literature. For example, the SUSY version of the Ma model is studied in e.g., [30, 31]. The SUSY version of the AKS model is provided as a low energy effective theory of a SUSY SU $(2)_{H}$ gauge theory with confinement, which is briefly introduced in section 3 .

\section{A UV PICTURE}

The models discussed in the previous section are interesting as phenomenological models, since several new particles are introduced at around the $\mathrm{TeV}$ scale so that many new phenomena are predicted and will be tested in future experiments. However, they seem to be artificial from a view point of a fundamental theory. Here we would like to consider a example UV picture of 
A

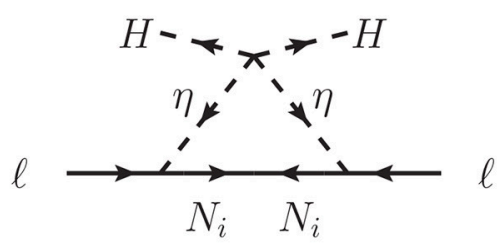

C

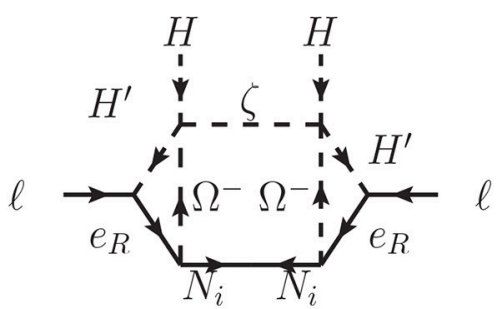

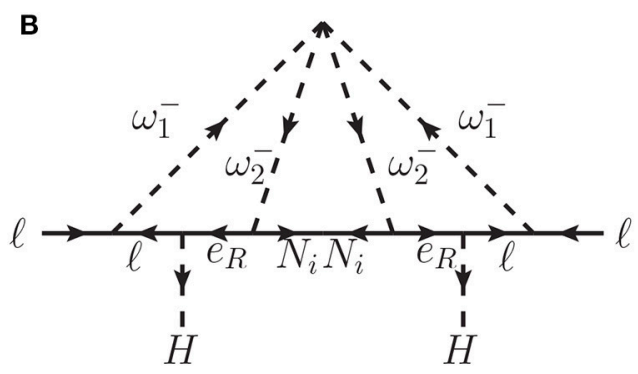

FIGURE 1 | Relevant diagrams for neutrino mass in (A) the Ma model [29], (B) the KNT model [28], and (C) the AKS model [32-34].

such a phenomenological model. In Kanemura et al. [49-52], an concrete example of UV theory of a loop induced neutrino mass model is proposed. The theory is based on a SUSY gauge theory with confinement.

In SUSY $\mathrm{SU}\left(N_{c}\right)$ gauge theory with $N_{c}+1$ flavor fields, it is known that confinement occurs at some scale [65]. We use this setup and we consider a model with $\mathrm{SU}(2)_{H}$ symmetry with three flavor fields. These three flavor fields are fundamental representations of $\mathrm{SU}(2)_{H}$. Note that each of three fields has their anti-matter partner so that there are six flavor fields in total. We describe these fields as $T_{i}(i=1, \cdots, 6)$. After confinement, we have fifteen mesonic fields $H_{i j} \sim T_{i} T_{j}$. The setup of this model is almost the same as in the minimal SUSY fat Higgs model [66]. In this model, additional fields are introduced in order to make only two doublets and one singlet mesonic fields light. In the model considered here, in contrast, all the mesonic fields appears in the low energy effective theory.

We here additionally introduce a RHN which is singlet under both $\mathrm{SU}(2)_{H}$ and the $\mathrm{SM}$ gauge symmetries. We assume that the model has an unbroken discrete symmetry $Z_{2}$ which forbids tree level contributions to neutrino masses. The RHN is considered as an $Z_{2}$ odd field. Table 1I shows the charge assignments of $T_{i}$ and the RHN $N_{R}^{c}$ under the SM gauge symmetry, $S U(2)_{H}$, and the $Z_{2}$ parity, and Table 1II shows the fifteen mesonic fields below the confinement scale $\Lambda_{H}$ which are canonically normalized as $H_{i j} \simeq \frac{1}{4 \pi \Lambda_{H}} T_{i} T_{j}(i \neq j)$.

The superpotential of the Higgs sector below $\Lambda_{H}$ is given by

$$
\begin{aligned}
W_{\mathrm{eff}} & =\lambda N\left(H_{u} H_{d}+v_{0}^{2}\right)+\lambda N_{\Phi}\left(\Phi_{u} \Phi_{d}+v_{\Phi}^{2}\right) \\
& +\lambda N_{\Omega}\left(\Omega_{+} \Omega_{-}-\zeta \eta+v_{\Omega}^{2}\right)+\lambda\left\{\zeta H_{d} \Phi_{u}+\eta H_{u} \Phi_{d}\right. \\
& \left.-\Omega_{+} H_{d} \Phi_{d}-\Omega_{-} H_{u} \Phi_{u}-N N_{\Phi} N_{\Omega}\right\}
\end{aligned}
$$

TABLE 1 | (I) The charge assignment of the $S U(2)_{H}$ doublets $T_{i}$ and the RHN $N_{R}^{C}$ under the electroweak gauge group $\left(S U(2) L \times U(1)_{Y}\right)$ and the $Z_{2}$ parity. (II) The field

\begin{tabular}{|c|c|c|c|c|}
\hline Superfield & $\mathrm{SU}(2)_{H}$ & $\mathrm{SU}(2)_{L}$ & $\mathrm{U}(1)_{Y}$ & $z_{2}$ \\
\hline \multicolumn{5}{|l|}{ (I) } \\
\hline$\left(\begin{array}{l}T_{1} \\
T_{2}\end{array}\right)$ & 2 & 2 & 0 & +1 \\
\hline$T_{3}$ & 2 & 1 & $+1 / 2$ & +1 \\
\hline$T_{4}$ & 2 & 1 & $-1 / 2$ & +1 \\
\hline$T_{5}$ & 2 & 1 & $+1 / 2$ & -1 \\
\hline$T_{6}$ & 2 & 1 & $-1 / 2$ & -1 \\
\hline$N_{R}^{C}$ & 1 & 1 & 0 & -1 \\
\hline \multicolumn{4}{|c|}{ Superfield } & $z_{2}$ \\
\hline \multicolumn{5}{|l|}{ (II) } \\
\hline & $\begin{array}{l}=\left(\begin{array}{l}H_{14} \\
H_{24}\end{array}\right. \\
H_{56}, N_{\Phi}\end{array}$ & $\begin{array}{r}=\left(\begin{array}{l}H_{13} \\
H_{23}\end{array}\right) \\
N_{\Omega}=H_{1}\end{array}$ & & +1 \\
\hline & $\left(\begin{array}{l}H_{15} \\
H_{25}\end{array}\right)$ & $\left(\begin{array}{l}H_{16} \\
H_{26}\end{array}\right)$ & & -1 \\
\hline \multicolumn{5}{|c|}{$\Omega_{-} \equiv H_{46}, \Omega_{+} \equiv H_{35}$} \\
\hline
\end{tabular}
content of the extended Higgs sector in the low energy effective theory below the scale $\Lambda_{H}$.

By the naive dimensional analysis, one expects $\lambda \simeq 4 \pi$ at the confinement scale $\Lambda_{H}$. We here assume that the mass parameters $\mu=\lambda\langle N\rangle, \mu_{\Phi}=\lambda\left\langle N_{\Phi}\right\rangle$ and $\mu_{\Omega}=\lambda\left\langle N_{\Omega}\right\rangle$ are induced by the vacuum expectation values (vev's) of $Z_{2}$-even singlet fields $N, N_{\Phi}$ and $N_{\Omega}$. The Yukawa couplings and the Majorana mass term of 
the RHNs are given by

$$
W_{N}=y_{N}^{i} N_{R}^{c} L_{i} \Phi_{u}+h_{N}^{i} N_{R}^{c} E_{i}^{c} \Omega_{-}+\frac{M_{R}}{2} N_{R}^{c} N_{R}^{c}+\frac{\kappa}{2} N N_{R}^{c} N_{R}^{c} .
$$

In the low energy effective theory of this model, the dimension five operator is generated via loop contributions shown in Figure 1 of Kanemura et al. [52] (one of the diagrams is shown in Figure 1C in this paper). There are both one-loop and three-loop contributions. The one-loop and three-loop diagrams correspond to the SUSY versions of the Ma model [29] and AKS model [32-34], respectively. It is worthwhile pointing out that the one-loop diagrams and three-loop diagrams are controlled by different coupling constants, i.e., one-loop diagrams are driven by the coupling $y_{N}$ and the three-loop diagrams are controlled by another coupling $h_{N}$. Both one-loop and threeloop contributions can be significant if these coupling constants are hierarchical as $h_{N} \gg y_{N}$. Therefore, two different mass squared differences can be generated even if only one RHN is introduced ${ }^{1}$.

In the $\mathrm{SU}(2)_{H}$ model, not only the tiny neutrino masses but also other unsolved problems in the SM such as DM and baryogenesis may be solved. For the DM, the model contains the unbroken $Z_{2}$ symmetry as well as the unbroken $R$ parity. These discrete symmetries guarantee the stability of DM candidates. Since there are two different unbroken parities, there are potentially three kinds of DM candidates, i.e., the lightest particles with the parity assignments of $(-,+),(+,-)$, and $(-,-)$. If we consider the case that one of them is heavier than the sum of the masses of the others, the heaviest one decays into the other two DM particles so that the heaviest particle cannot be a DM.

Also, electroweak baryogenesis may work in the $\mathrm{SU}(2)_{H}$ model. It is known that for successful electroweak baryogenesis, the 1st order phase transition (1stOPT) should be strong enough. This condition can be described by the inequality $\varphi_{c} / T_{c}>$ 1. In addition, new $\mathrm{CP}$ violation phases are required in order to reproduce the correct amount of baryon asymmetry of the Universe. In this model, the 1stOPT can be enhanced by loop contributions of extra $Z_{2}$-odd scalar particles strongly enough. Though the analysis on $\mathrm{CP}$ phases in this model has not been done yet, it is naively expected that we can introduce several CP phases relevant to baryogenesis as in the case of $\operatorname{MSSM}[67,68]$.

In Kanemura et al. [52], a benchmark scenario is provided. It reproduces the appropriate neutrino mass matrix, explains the DM relic abundance, and satisfies the 1stOPT condition as well as the constraints from the experimental data such as from lepton flavor violating processes searches. In Figure 3 of Kanemura et al. [52], the mass spectrum of the relevant particles in this benchmark scenario is shown.

The benchmark parameter point discussed above is already excluded by the direct detection experiment of the DM [69]. However, the predicted spin independent cross section can be

\footnotetext{
${ }^{1}$ In the ordinary type-I seesaw model, at least two RHNs are necessary for generating two different mass squared differences.
}

significantly smaller, if we take into account the CP phases [70]. It is because the pseudo-scalar interaction with DM fermions are not relevant to the spin-independent cross section. Such $\mathrm{CP}$ phases can affect the BAU. Therefore, it may be interesting to discuss the correlation among BAU, spin-independent cross section, and other $\mathrm{CP}$ violating phenomena such as electric dipole moments of electron, neutron, and so on. This kind of analysis remains as a future task.

We here discuss phenomenological consequences of the benchmark scenario. The $Z_{2}$-even part of the spectrum is similar to the one in the nMSSM. In order to reproduce the relic abundance of the DM, a large mixing between doublet fields and singlet scalars are required. As a consequence, large mass splitting between the charged Higgs boson and the heavy Higgs bosons is predicted. The $Z_{2}$-even part of this scenario can be distinguished from the MSSM by looking at such a specific mass spectrum.

The condition $\varphi_{c} / T_{c}>1$ is satisfied by loop effects of $\Phi_{u}$ and $\Omega_{-}$. The same scalars also significantly affect the SM-like Higgs boson couplings, especially, the $h-\gamma-\gamma$ coupling and the triple Higgs boson coupling. The prediction on the deviation of the SM-like Higgs couplings in this benchmark scenario is given by

$$
\begin{aligned}
\kappa_{h W W} & =0.990, \quad \kappa_{h Z Z}=0.990, \quad \kappa_{h \bar{u} u}=0.990 \\
\kappa_{h \bar{d} d} & =0.978, \quad \kappa_{h \bar{\ell} \ell}=0.978 \\
\kappa_{h \gamma \gamma} & =0.88, \quad \kappa_{h h h}=1.2
\end{aligned}
$$

where the $\kappa$ 's denote the ratios between coupling constants predicted in this benchmark point and ones predicted in the SM, i.e.,

$$
\kappa_{h \phi \phi}=\frac{g_{h \phi \phi}}{g_{h \phi \phi}^{S M}} .
$$

Here, in particular, the deviations in $h \rightarrow \gamma \gamma$ and the self coupling constant of the Higgs boson are as significant as $10-20 \%$. By precise measurements of the SM-like Higgs boson couplings at future collider experiment such as the ILC [71, 72], the model can be distinguished from the nMSSM too.

Let us consider the $Z_{2}$-odd sector. By direct search for inert doublet particles [73] and inert charged singlet searches [74] at a lepton collider, we expect to get a strong hint on the $Z_{2}$-odd sector of the scenario.

\section{SUMMARY}

We have reviewed some models with loop induced neutrino masses. Although such models are phenomenologically quite interesting, they seems to be artificial. We have discussed an example based on SUSY $\mathrm{SU}(2)_{H}$ gauge theory with confinement as a UV picture of such a phenomenological model. In the low energy effective theory, three problems in the SM namely baryogenesis, DM, and tiny neutrino mass can be solved. The 1stOPT is enhanced strongly enough for successful electroweak baryogenesis [53-61], multi-components 
DM scenario is realized, and tiny neutrino masses [23-34] are generated via one-loop and three-loop diagrams. This model has a big advantage over the canonical type-I seesaw model. It is that new particles are required at a few $\mathrm{TeV}$ range so that the model will be tested at future experiments.

In models where tiny neutrino masses are radiatively generated via loop diagrams, the Higgs sector is often extended by introducing additional scalar fields. These additional scalar fields can contribute to various phenomenology. Some models can be distinguished with use of patterns of various phenomenological signals which are expected to be measured in future experiments. Then, it is expected that a UV theory which leads to a model with loop induced neutrino masses can be explored by investigating a pattern of various experimental

\section{REFERENCES}

1. Aad G, Bentvelsenet S, Berglund E, Bobbink GJ, Bos K, Boterenbrood H, et al. Combined search for the Standard Model Higgs boson using up to $4.9 \mathrm{fb}^{-1}$ of $p p$ collision data at $\sqrt{s}=7 \mathrm{TeV}$ with the ATLAS detector at the LHC. Phys Lett. (2012) B710:49-66. doi: 10.1016/j.physletb.2012.02.044

2. Chatrchyan S, Khachatryanet V, Sirunyan A, Tumasyan A, Adam W, Bergaueral $\mathrm{T}$, et al. Combined results of searches for the standard model Higgs boson in $p p$ collisions at $\sqrt{s}=7 \mathrm{TeV}$. Phys Lett. (2012) B710:26-48. doi: 10.1016/j.physletb.2012.02.064

3. Aad G, Abbott B, Abdallah J, Abdinov O, Aben R, Abolins M, et al. Study of the spin and parity of the Higgs boson in diboson decays with the ATLAS detector. Eur Phys J. (2015) C75:476. [Erratum: Eur. Phys. J.C76,no.3,152(2016)]. doi: 10.1140/epjc/s10052-015-3685-1

4. Khachatryan V, Apresyan A, Bornheim A, Bunn J, Chen Y, Duarte J, et al. Constraints on the spin-parity and anomalous HVV couplings of the Higgs boson in proton collisions at 7 and $8 \mathrm{TeV}$. Phys Rev. (2015) D92:012004. doi: 10.1103/PhysRevD.92.012004

5. Aad G, Abbott B, Abdallah J, Abdinov O, Aben R, Abolins M, et al. Measurements of the Higgs boson production and decay rates and coupling strengths using pp collision data at $\sqrt{s}=7$ and $8 \mathrm{TeV}$ in the ATLAS experiment. Eur Phys J. (2016) C76:6. doi: 10.1140/epjc/s10052-015-3769-y

6. Khachatryan V, Sirunyan AM, Tumasyan A, Adam W, Bergauer T, Dragicevic $\mathrm{M}$, et al. Precise determination of the mass of the Higgs boson and tests of compatibility of its couplings with the standard model predictions using proton collisions at 7 and 8 TeV. Eur Phys J. (2015) C75:212. doi: 10.1140/epjc/s10052-015-3351-7

7. Fukuda Y,Hayakawa T, Ichihara E, Inoue K, Ishihara K, Ishino $\mathrm{H}$, et al. Evidence for oscillation of atmospheric neutrinos. Phys Rev Lett. (1998) 81:1562-7. doi: 10.1103/PhysRevLett.81.1562

8. Ahmad QR, Allen RC, Andersen TC, Anglin DJ, Barton JC, Beier EW, et al. Direct evidence for neutrino flavor transformation from neutral current interactions in the Sudbury Neutrino Observatory. Phys Rev Lett. (2002) 89:011301. doi: 10.1103/PhysRevLett.89.011301

9. Eguchi K, Enomoto S, Furuno K, Goldman J, Hanada H, Ikeda H, et al. First results from KamLAND: evidence for reactor anti-neutrino disappearance. Phys Rev Lett. (2003) 90:021802. doi: 10.1103/PhysRevLett.90.021802

10. An FP, Bai JZ, Balantekin AB, Band HR, Beavis D, Beriguete W, et al. Observation of electron-antineutrino disappearance at Daya Bay. Phys Rev Lett. (2012) 108:171803. doi: 10.1103/PhysRevLett.108.171803

11. Ahn JK, Chebotaryov S, Choi JH, Choi S, Choi W, Choi Y, et al. Observation of reactor electron antineutrino disappearance in the RENO experiment. Phys Rev Lett. (2012) 108:191802. doi: 10.1103/PhysRevLett.108.191802

12. Abe K, Adam J, Aihara H, Akiri T, Andreopoulos C, Aoki S, et al. Observation of electron neutrino appearance in a muon neutrino beam. Phys Rev Lett. (2014) 112:061802. doi: 10.1103/PhysRevLett.112.061802

13. Otten EW, Weinheimer C. Neutrino mass limit from tritium beta decay. Rept Prog Phys. (2008) 71:086201. doi: 10.1088/0034-4885/71/8/086201 signals. This situation is very different from a case of a grand unified theory with a grand desert such as SUSY SU(5) GUT.

\section{AUTHOR CONTRIBUTIONS}

The author confirms being the sole contributor of this work and has approved it for publication.

\section{ACKNOWLEDGMENTS}

This work was supported by JSPS KAKENHI Grant Number 17H05408 and by the Kogakuin University Grant for the project research.

14. Drexlin G, Hannen V, Mertens S, Weinheimer C. Current direct neutrino mass experiments. Adv High Energy Phys. (2013) 2013:293986. doi: $10.1155 / 2013 / 293986$

15. Weinberg S. Baryon and lepton nonconserving processes. Phys Rev Lett. (1979) 43:1566-70. doi: 10.1103/PhysRevLett.43.1566

16. Minkowski P. $\mu \rightarrow e \gamma$ at a rate of one out of $10^{9}$ muon decays? Phys Lett. (1977) 67B:421-8.

17. Yanagida T. KEK report KEK-79-18. In: O. Sawada and A. Sugamoto, editors. Workshop on the Unified Theory and the Baryon Number in the Universe, Tsukuba (1979). p. 95.

18. Yanagida T. Horizontal symmetry and masses of neutrinos. Prog Theor Phys. (1980) 64:1103.

19. Gell-Mann M, Ramond P, Slansky R. Complex spinors and unified theories. Conf Proc. (1979) C790927:315-21.

20. Mohapatra RN, Senjanovic G. Neutrino mass and spontaneous parity violation. Phys Rev Lett. (1980) 44:912

21. Mohapatra RN. Mechanism for understanding small neutrino mass in superstring theories. Phys Rev Lett. (1986) 56:561-3.

22. Mohapatra RN, Valle JWF. Neutrino mass and Baryon number nonconservation in superstring models. Phys Rev. (1986) D34:1642.

23. Zee A. A theory of Lepton number violation, neutrino Majorana mass, and oscillation. Phys Lett. (1980) 93B:389. [Erratum: Phys. Lett.95B,461(1980)].

24. Kanemura S, Shindou T, Sugiyama H. R-parity conserving supersymmetric extension of the Zee model. Phys Rev. (2015) D92:115001. doi: 10.1103/PhysRevD.92.115001

25. Zee A. Quantum numbers of Majorana neutrino masses. Nucl Phys. (1986) B264:99-110.

26. Babu KS. Model of 'Calculable' Majorana neutrino masses. Phys Lett. (1988) B203:132-6.

27. Aoki M, Kanemura S, Shindou T, Yagyu K. An R-parity conserving radiative neutrino mass model without right-handed neutrinos. JHEP. (2010) 7:084. [Erratum: JHEP11,049(2010)].doi: 10.1007/JHEP07(2010)084

28. Krauss LM, Nasri S, Trodden M. A model for neutrino masses and dark matter. Phys Rev. (2003) D67:085002. doi: 10.1103/PhysRevD.67.085002

29. Ma E. Verifiable radiative seesaw mechanism of neutrino mass and dark matter. Phys Rev. (2006) D73:077301. doi: 10.1103/PhysRevD.73.077301

30. Ma E. Supersymmetric model of radiative Seesaw Majorana neutrino masses. Annales FondBroglie. (2006) 31:285.

31. Ma E. Supersymmetric U(1) gauge realization of the dark scalar doublet model of radiative neutrino mass. Mod Phys Lett. (2008) A23:721-5. doi: 10.1142/S0217732308026753

32. Aoki M, Kanemura S, Seto O. Neutrino mass, dark matter and Baryon asymmetry via TeV-scale physics without fine-tuning. Phys Rev Lett. (2009) 102:051805. doi: 10.1103/PhysRevLett.102.051805

33. Aoki M, Kanemura S, Seto O. A model of $\mathrm{TeV}$ scale physics for neutrino mass, dark matter and Baryon asymmetry and its phenomenology. Phys Rev. (2009) D80:033007. doi: 10.1103/PhysRevD.80. 033007 
34. Aoki M, Kanemura S, Yagyu K. Triviality and vacuum stability bounds in the three-loop neutrino mass model. Phys Rev. (2011) D83:075016. doi: 10.1103/PhysRevD.83.075016

35. Cai Y, Herrero-García J, Schmidt MA, Vicente A, Volkas RR. From the trees to the forest: a review of radiative neutrino mass models. Front Phys. (2017) 5:63. doi: 10.3389/fphy.2017.00063

36. Nomura T, Okada H, Okada N. A colored KNT neutrino model. Phys Lett. (2016) B762:409-14. doi: 10.1016/j.physletb.2016.09.038

37. Cheung $K$, Nomura $T$, Okada $H$. Three-loop neutrino mass model with a colored triplet scalar. Phys Rev. (2017) D95:015026. doi: 10.1103/PhysRevD.95.015026

38. Doršner I, Fajfer S, Faroughy DA, Košnik N. The role of the $S_{3}$ GUT leptoquark in flavor universality and collider searches. J High Energ Phys. (2017) 2017: 188. doi: 10.1007/JHEP10(2017)188

39. Guo SY, Han ZL, Li B, Liao Y, Ma XD. Interpreting the $R_{K^{(*)}}$ anomaly in the colored Zee-Babu model. Nucl Phys. (2018) B928:435-47. doi: 10.1016/j.nuclphysb.2018.01.024

40. Hati C, Kumar G, Orloff J, Teixeira AM. Reconciling B-meson decay anomalies with neutrino masses, dark matter and constraints from flavour violation. JHEP. (2018) 11:011. doi: 10.1007/JHEP11(2018)011

41. Lees JP, Chaoet DT, Cheng CH, Echenard B, Floodal KT, Hitlin D, et al. Measurement of an excess of $\bar{B} \rightarrow D^{(*)} \tau^{-} \bar{\nu}_{\tau}$ decays and implications for charged Higgs bosons. Phys Rev. (2013) D88:072012. doi: 10.1103/PhysRevD.88.072012

42. Aaij R, Anderson J, Bernet R, Bowen E, Bursche A, Chiapolini $\mathrm{N}$, et al. Measurement of the ratio of branching fractions $\mathcal{B}\left(\bar{B}^{0} \rightarrow D^{*+} \tau^{-} \bar{v}_{\tau}\right) / \mathcal{B}\left(\bar{B}^{0} \rightarrow D^{*+} \mu^{-} \bar{v}_{\mu}\right)$. Phys Rev Lett. (2015) 115:111803. [Erratum: Phys. Rev. Lett.115,no.15,159901(2015)]. doi: 10.1103/PhysRevLett.115.111803

43. Fajfer S, Kamenik JF, Nisandzic I. On the $B \rightarrow D^{*} \tau \bar{\nu}_{\tau}$ sensitivity to new physics. Phys Rev. (2012) D85:094025. doi: 10.1103/PhysRevD.85.094025

44. Bernlochner FU, Ligeti Z, Papucci M, Robinson DJ. Combined analysis of semileptonic $B$ decays to $D$ and $D^{*}: R\left(D^{(*)}\right),\left|V_{c b}\right|$, and new physics. Phys Rev. (2017) D95:115008. [Erratum: Phys. Rev.D97,no.5,059902(2018)]. doi: 10.1103/PhysRevD.97.059902

45. Bigi D, Gambino P, Schacht S. A fresh look at the determination of $\left|V_{c b}\right|$ from $B \rightarrow D^{*} \ell$ v. Phys Lett. (2017) B769:441-5. doi: 10.1016/j.physletb.2017.04.022

46. Hiller G, Kruger F. More model-independent analysis of $b \rightarrow s$ processes. Phys Rev. (2004) D69:074020. doi: 10.1103/PhysRevD.69.074020

47. Bordone $\mathrm{M}$, Isidori G, Pattori A. On the standard model predictions for $R_{K}$ and $R_{K^{*}}$. Eur Phys J. (2016) C76:440. doi: 10.1140/epjc/s10052-0164274-7

48. Doršner I, Fajfer S, Košnik N. Leptoquark mechanism of neutrino masses within the grand unification framework. Eur Phys J. (2017) C77:417. doi: 10.1140/epjc/s10052-017-4987-2

49. Kanemura S, Machida N, Shindou T, Yamada T. A UV complete model for radiative seesaw scenarios and electroweak baryogenesis based on the supersymmetric gauge theory. Phys Rev. (2014) D89:013005. doi: 10.1103/PhysRevD.89.013005

50. Kanemura S, Senaha E, Shindou T, Yamada T. Electroweak phase transition and Higgs boson couplings in the model based on supersymmetric strong dynamics. J High Energy Phys. (2013) 5:66.

51. Kanemura S, Shindou T, Yamada T. A light Higgs scenario based on the TeV-scale supersymmetric strong dynamics. Phys Rev. (2012) D86:55023. doi: 10.1103/PhysRevD.86.055023

52. Kanemura S, Machida N, Shindou T. Radiative neutrino mass, dark matter and electroweak baryogenesis from the supersymmetric gauge theory with confinement. Phys Lett. (2014) B738:178-86. doi: 10.1016/j.physletb.2014.09.013

53. Kuzmin VA, Rubakov VA, Shaposhnikov ME. On the anomalous electroweak Baryon number nonconservation in the early universe. Phys Lett. (1985) 155B:36. doi: 10.1016/0370-2693(85)91028-7

54. Cohen AG, Kaplan DB, Nelson AE. Progress in electroweak baryogenesis. Ann Rev Nucl Part Sci. (1993) 43:27-70. doi: 10.1146/annurev.ns.43.120193.000331

55. Quiros M. Field theory at finite temperature and phase transitions. Helv Phys Acta. (1994) 67:451-583.
56. Rubakov VA, Shaposhnikov ME. Electroweak baryon number nonconservation in the early universe and in high-energy collisions. Usp Fiz Nauk. (1996) 166:493-537. [Phys. Usp.39,461(1996)].

57. Funakubo K. CP violation and baryogenesis at the electroweak phase transition. Prog Theor Phys. (1996) 96:475-520.

58. Trodden M. Electroweak baryogenesis. Rev Mod Phys. (1999) 71:1463-500.

59. Bernreuther W. CP violation and baryogenesis. Lect Notes Phys. (2002) 591:237-93.

60. Cline JM. Baryogenesis. In: Les Houches Summer School-Session 86: Particle Physics and Cosmology: The Fabric of Spacetime Les Houches. Paris (2006).

61. Morrissey DE, Ramsey-Musolf MJ. Electroweak baryogenesis. New J Phys. (2012) 14:125003. doi: 10.1088/1367-2630/14/12/125003

62. Aoki M, Kubo J, Takano H. Two-loop radiative seesaw mechanism with multicomponent dark matter explaining the possible $\gamma$ excess in the Higgs boson decay and at the Fermi LAT. Phys Rev. (2013) D87:116001. doi: 10.1103/PhysRevD.87.116001

63. Kajiyama Y, Okada H, Yagyu K. Two loop radiative seesaw model with inert triplet scalar field. Nucl Phys. (2013) B874:198-216. doi: 10.1016/j.nuclphysb.2013.05.020

64. Aoki M, Kanemura S, Tsumura K, Yagyu K. Models of Yukawa interaction in the two Higgs doublet model, and their collider phenomenology. Phys Rev. (2009) D80:15017. doi: 10.1103/PhysRevD.80.0 15017

65. Intriligator KA, Seiberg N. Lectures on supersymmetric gauge theories and electric-magnetic duality. Nucl Phys Proc Suppl. (1996) 45BC:1-28. doi: 10.1016/0920-5632(95)00626-5

66. Harnik R, Kribs GD, Larson DT, Murayama H. The Minimal supersymmetric fat Higgs model. Phys Rev. (2004) D70:15002. doi: 10.1103/PhysRevD.70.015002

67. Dine M, Huet P, Singleton RL Jr, Susskind L. Creating the baryon asymmetry at the electroweak phase transition. Phys Lett. (1991) B257:351-56. doi: 10.1016/0370-2693(91)91905-B

68. Cohen AG, Nelson AE. Supersymmetric baryogenesis. Phys Lett. (1992) B297:111-7.

69. Akerib DS, Alsum S, Araújo HM, Bai X, Bailey AJ, Balajthy J, et al. Results from a search for dark matter in the complete LUX exposure. Phys Rev Lett. (2017) 118:021303. doi: 10.1103/PhysRevLett.118. 021303

70. Abe T. Effect of CP violation in the singlet-doublet dark matter model. Phys Lett. (2017) B771:125-30. doi: 10.1016/j.physletb.2017. 05.048

71. Baer H, Barklow T, Fujii K, Gao Y, Hoang A, Kanemura S, et al. The international linear collider technical design report - volume 2: physics. arXiv:1306.6352 (2013).

72. Asner DM, Barklow T, Calancha C, Fujii K, Graf N, Haber HE, et al. ILC Higgs White Paper. In: Proceedings, 2013 Community Summer Study on the Future of U.S. Particle Physics Minneapolis, MN:Snowmass on the Mississippi (CSS2013) (2013). Available online at: http://www.slac.stanford.edu/econf/ C1307292/docs/submittedArxivFiles/1310.0763.pdf

73. Aoki M, Kanemura S, Yokoya H. Reconstruction of inert doublet scalars at the international linear collider. Phys Lett. (2013) B725:302-9. doi: 10.1016/j.physletb.2013.07.011

74. Aoki M, Kanemura S. Probing the Majorana nature of TeV-scale radiative seesaw models at collider experiments. Phys Lett. (2010) B689:28-35. doi: 10.1016/j.physletb.2010.04.024

Conflict of Interest Statement: The author declares that the research was conducted in the absence of any commercial or financial relationships that could be construed as a potential conflict of interest.

Copyright (C) 2019 Shindou. This is an open-access article distributed under the terms of the Creative Commons Attribution License (CC BY). The use, distribution or reproduction in other forums is permitted, provided the original author(s) and the copyright owner(s) are credited and that the original publication in this journal is cited, in accordance with accepted academic practice. No use, distribution or reproduction is permitted which does not comply with these terms. 